\title{
Reconciling the Cosmological Constant with the Energy Density of Quantum Field Theories of the Zeropoint
}

\author{
Remi Cornwall \\ University of London Alumni Society, Malet Street, London WC1E 7HU \\ http://www.evosci.org http://ulondon.academia.edu/RemiCornwall
}

\begin{abstract}
This paper results from our investigation into novel means of electromagnetic propulsion. It requires the basis of our claims to be put on a sound theoretical footing regarding the purported momentum exchange with the electromagnetic field. One of these concerns is the huge discrepancy between the energy density of the Zeropoint and its purported manifestation as the Cosmological Constant. Here we state that it is manifestly wrong to introduce the zeropoint at zero order into the stress-energy tensor, because it is something which describes zero particle count. As a fluctuation, it belongs in a higher order Taylor expansion in frequency of the stress-energy tensor. Furthermore in the $3^{\text {rd }}$ order in the Einstein constant our procedure is some 9 orders of magnitude too small. We make up this difference by suggesting that vacuum energy is much higher still and that more degrees of freedom exist in physics beyond the Standard Model or that there is interaction energy between the modes.
\end{abstract}

Keywords: Zeropoint Energy, Cosmological Constant, Stress Energy Tensor, Einstein Field Equations, Standard Model, Dark Energy, Quantum Gravity.

\section{$\underline{\text { 1. Introduction }}$}

We have been seeking to put a putative electromagnetic propulsor[1], which is based on the Feynman/Heaviside Disk/static field momentum conjecture[2-6], on a sound theoretical footing[7-9] and as such, believe it viable to talk about "dumping" momentum to the "zeropoint" of the electromagnetic field. We are in the process of trying to show how the zeropoint behaves like a superfluid or supersolid with mechanical properties, such as the ability propagate waves and thermalize momentum imparted to it from the propulsor. The effect of zeropoint fluctuations is not contested, it is behind the physics of spontaneous emission, the Lamb Shift, Van der Waals forces[10] but short of new physics to explain Dark Energy and universal expansion, the zeropoint is seen as the explanation for this... save a huge difference[11] in the magnitude of this energy density compared to the cosmological constant ${ }^{\dagger}$. Understanding this has then fallen within the remit of the electromagnetic propulsion project.

Particle physics has been described as ever more cunning applications of the quantised harmonic oscillator[12]; the basic Hamiltonians of quantised harmonic oscillators for boson and fermion fields with their ladder operators are listed here:-

$$
H_{B}=\frac{1}{2} \hbar \omega\left(a^{\dagger} a+a a^{\dagger}\right)=\left(a^{\dagger} a+\frac{1}{2}\right) \hbar \omega \text { eqn. } 1
$$

$\rho_{Q F T} \sim 10^{113} \mathrm{~J} / \mathrm{m}^{3}, \rho_{C C} \sim 10^{-9} \mathrm{~J} / \mathrm{m}^{3}$ respectively.

$$
H_{F}=\frac{1}{2} \hbar \omega\left(b^{\dagger} b-b b^{\dagger}\right)=\left(b^{\dagger} b-\frac{1}{2}\right) \hbar \omega \text { eqn. } 2
$$

Quantum mechanics involves differences in energy, so the zeropoint terms don't matter, even then there is normal ordering[10] to remove these terms. However in General Relativity it would seem that the absolute value of the energy density of these fields is relevant by the central equation,

$$
\mathbf{R}_{\mu v}-\frac{1}{2} R \mathbf{g}_{\mu v}=\frac{8 \pi G}{c^{4}} \mathbf{T}_{\mu v} \quad \text { eqn. } 3
$$

And this energy density is of the order[13, 14],

$$
\begin{aligned}
& \rho_{0, \text { boson }}=\frac{\hbar}{2}\left(\frac{1}{2 \pi}\right)^{3} \int_{0}^{K} k^{2} \sqrt{k^{2}+m^{2}} d k \\
& \rho_{0, \text { fermion }}=-2 \frac{\hbar}{2}\left(\frac{1}{2 \pi}\right)^{3} \int_{0}^{K} k^{2} \sqrt{k^{2}+m^{2}} d k
\end{aligned}
$$

Where $\mathrm{K}$ is a momentum cut-off. If the masses are neglected, the integrals can be estimated by integrating to the Planck Frequency:-

$$
\rho_{0}=\frac{\hbar}{8 \pi^{2} c^{3}} \int_{0}^{\omega_{p}} \omega^{3} d \omega \quad \text { eqn. } 5
$$

And this figure is huge, of the order of $10^{113} \mathrm{~J} / \mathrm{m}^{3}$ (later we'll argue it should be higher still). Pauli[13] argued by relativistic invariance of the ideal gas representing the zeropoint, that there would be a momentum cut-off thus,

$$
\int_{0}^{K} k^{2} \sqrt{k^{2}+m^{2}} d k=\frac{K^{4}}{4}+\frac{m^{2} K^{2}}{4}-\frac{m^{4}}{4} \log \frac{2 K}{m}+O\left(\frac{1}{K}\right)
$$
eqn. 6 
And then sought to cancel the positive zeropoint of boson fields against the negative zeropoint of fermion fields by the constraint that the number of types of fermion particles is twice that of boson particles (factor of 2 in fermion contribution eqn. 4) by these requirements

$$
\begin{aligned}
& \sum_{i}\left(m_{0}^{i}\right)^{2}=2 \sum_{j}\left(m_{1 / 2}^{j}\right)^{2} \\
& \sum_{i}\left(m_{0}^{i}\right)^{4}=2 \sum_{j}\left(m_{1 / 2}^{j}\right)^{4} \\
& \sum_{i}\left(m_{0}^{i}\right)^{4} \log m_{0}^{i}=2 \sum_{j}\left(m_{1 / 2}^{j}\right)^{4} \log m_{1 / 2}^{j}
\end{aligned}
$$

The zero mass of the photon of the electromagnetic field would dominate the LHS; such a cancellation is impossible and the zeropoint is of the order given by eqn. 5 .

The homogenous, isotropic zeropoint, with no preferred frame, is represented in the stress-energy tensor as an ideal fluid with $\rho_{0}=-p$,

$$
\mathbf{T}_{\mu \nu}=\left(\begin{array}{cccc}
\rho_{0} & 0 & 0 & 0 \\
0 & -p & 0 & 0 \\
0 & 0 & -p & 0 \\
0 & 0 & 0 & -p
\end{array}\right)
$$

The trace of the tensor is,

$$
\rho_{0}-3 p=-2 p
$$

The units of the LHS of the Einstein Field Equations (EFEs, eqn. 3) are $\mathrm{m}^{-2}$ and a contraction on both indices gives:

$$
R=-\frac{8 \pi G}{c^{4}} T
$$

The scalar curvature, $\mathrm{R}$, is basically saying that a sphere of dust around a gravitating source would decrease in volume; specifically if the positive mass-energy in the $\mathrm{T}_{00}>\sum \mathrm{T}_{\mathrm{ii}}$ terms on the RHS, it would cause the surface area and volume to decrease (eqn. 9). Conversely, in a region where the negative pressure of the zeropoint dominates positive mass-energy, space would expand and that is exactly what we observe in Hubble expansion of the Universe.

\section{A Glaring Error in current approaches and then some Numerological Speculation}

The huge disparity (at least $10^{120}$ ) between the Cosmological Constant and the vacuum energy density of quantum field theories (QFT) has been

\footnotetext{
$\doteqdot$ Visser[14] shows the above as a direct consequence of Lorentz invariance, the finiteness of the zeropoint arises directly from this without a momentum cut-off.
}

described as the most embarrassing in physics, if not all of science. The cancellation program discussed in the introduction seems doomed to fail, so we must embrace the huge vacuum energy and somehow reconcile it with the absolute energy requirements of General Relativity.

However, if the stress-energy tensor of a particle moving along a trajectory $\mathbf{x}_{t r a j}(t)$ is given $b^{\S}$,

$$
\begin{aligned}
& \mathbf{T}_{\mu \nu}(\mathbf{x}, t)=\frac{E}{c^{2}} v_{\mu} v_{v} \delta\left(\mathbf{x}-\mathbf{x}_{\text {traj }}(t)\right) \\
& \text { with } v_{\mu}=\left(1, \frac{d \mathbf{x}_{t r a j}(t)}{d t}\right)
\end{aligned}
$$

then it is a glaring error by the research community to include expressions for vacuum energy densities in the zeroth order in the stress-energy tensor (density) - it makes no sense representing something that has zero particle count, unlike eqn. 11 where $E=n \hbar \omega$ is implicit for quanta of particles. It is only correct to consider the zeropoint as a fluctuation - it has variance but no average. We should write the stress-energy tensor as a Taylor series somehow in particle number and frequency. As the electromagnetic zeropoint will dominate, which was discussed towards the end of the introduction, eqn. 5 shall be used.

A few considerations make the process of writing the Taylor series even easier: space is approximately flat away from gravitating sources so we don't need the covariant derivative, we don't need to take the derivative with-respect-to the wave-4-vector either, as eqn. 5 has no wave-vector terms and so we can differentiate solely by frequency. Thus LHS of eqn. 3 is represented by $\mathbf{E}_{\mu \nu}(\omega), \kappa=\frac{8 \pi G}{c^{4}}$ and some mystery term $\mathrm{U}$ (to be discussed) so,

$$
\begin{aligned}
& \mathbf{E}_{\mu \nu}(\omega) U^{0}=\left.\mathbf{E}_{\mu \nu}(\omega)\right|_{0} U^{0}+U^{-1} \frac{d \mathbf{E}_{\mu \nu}(\omega)}{d \omega} \Delta \omega+U^{-2} \frac{1}{2} \frac{d^{2} \mathbf{E}_{\mu \nu}(\omega)}{d \omega^{2}}(\Delta \omega)^{2}+\ldots \\
& \Rightarrow \\
& \mathbf{E}_{\mu \nu}(\omega) U^{0}=\kappa U^{0} \mathbf{T}_{\mu \nu}(\omega)+\kappa^{2} U^{-1} \frac{d \mathbf{T}_{\mu \nu}(\omega)}{d \omega} \Delta \omega+\frac{1}{2} U^{-2} \kappa^{3} \frac{d^{2} \mathbf{T}_{\mu \nu}(\omega)}{d \omega^{2}}(\Delta \omega)^{2}+\ldots \\
& \text { eqn. } 12
\end{aligned}
$$

Our motivation is the purely numerical observation that the magnitude of $\left|\kappa^{3} \rho_{Q F T}\right|_{\text {magn }}$ is in the ballpark of $\rho_{c c}$.

\footnotetext{
${ }^{\S}$ Note this is not a tensor density as per EFE. We are interested in the form and appeal by analogy here.
} 
The zeroth order term (in $E_{\mu v}(\omega)$ ) on the RHS in regards to our cosmological constant problem is properly zero. The first order term would sum over positive and negative frequencies such that $\Delta \omega=0$ and the first order term is zero too (it could also be quashed by $\mathrm{U}$ ). This follows for every even power of $\Delta \omega$ too.

The $2^{\text {nd }}$ order term is the variance in the fluctuation of the zeropoint and $(\Delta \omega)^{2}>0$, which by the Energy-time Uncertainty Principle is easily calculated to be of the order of the Planck frequency, so nothing changes from eqn. 5 . However, what is U?

1. A device to mop up dimensional slackness $\left(\mathrm{U}^{-1}\right.$ units $\mathrm{N}$ or $\left.\mathrm{J} / \mathrm{m}\right)$.

2. A device that raised to power of zero has no effect on traditional EFE and brings in particle number in the zeroth order term.

3. A device to knock out the 1 st power term in $\kappa$ (though this can be quashed with our argument about $\pm \Delta \omega$ too).

4. A device that makes the fluctuation of the zeropoint relevant in 3 rd power of $\kappa\left(2^{\text {nd }}\right.$ order in $\mathbf{T}$ ) and ignores particle number count on all terms but the zeroth order (as already mentioned).

5. A device to fix $U^{-3} \kappa^{3} \rho_{Q F T}=\rho_{c c}$ (alternatively make $\rho_{Q F T}$ even bigger).

6. A scalar, a scaling of the identity matrix, a tensor, an operator?

Appendix 1 interprets what $U$ could be but we will run with the idea for now and the notion that the zeropoint might be some $10^{9}$ times bigger - it certainly shouldn't be present in the EFE stressenergy tensor (as it is wanted at present) to the zeroth order, so what harm is there in suggesting it has a different value?

\section{Is the Zeropoint much bigger?}

We have seen that between the zeropoint energies of the bosonic and fermionic fields that the bosonic dominates (eqn. 5 and eqn. 6) and could be the source of dark energy responsible for universal expansion. Section 2 concluded that, if our approach is correct, it should be some $10^{9}$ orders of magnitude higher still, maybe a factor of 1000 per spatial dimension. Where could this enter? New massive and hence short-ranged fields would be out of the question, as already discussed, for reasons of mass (eqn. 6). We might then look to physics beyond the Standard Model[12]; perhaps a $5^{\text {th }}$ long ranged force exists? Then, Grand Unified Theories (GUTs) suggest running coupling constants merge down from around 100 Planck lengths (figure 1), so perhaps down from there is the realm of String Theory. The contribution of the missing $10^{9}$ might be found there.

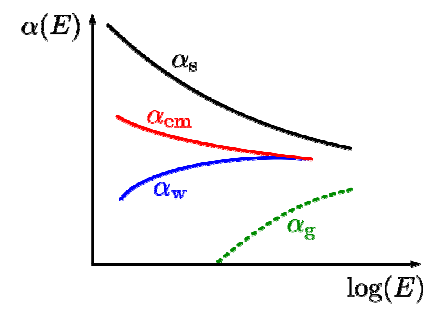

Figure 1 - Running coupling constants $\underline{\text { merging in GUTs }}$

For instance, String Theory[12] says there are 11 dimensions: 1 time and 10 spatial dimensions: 3 space, 7 compactified. This is approximately 1 spatial dimension +2 compactified per spatial dimension. We have $10^{9}$ to make up, so the deficit might be made up in those degrees of freedom. Most of the zeropoint energy arises at small length scales.

We have an alternative suggestion: although eqn. 5 is calculated assuming free fields, there might be interaction between the zeropoint modes such that the zeropoint becomes:

$$
H_{z p e}=\frac{1}{2}\left(\hbar \omega_{1}+\hbar \int I\left(\omega_{1}, d \omega\right) d \omega\right) \text { eqn. } 13
$$

The suggestion is that the mode $\omega_{1}$ in question is somehow convolved with the rest of the fluctuations from the other modes with an interaction term. Let us look into how this interaction term might arise.

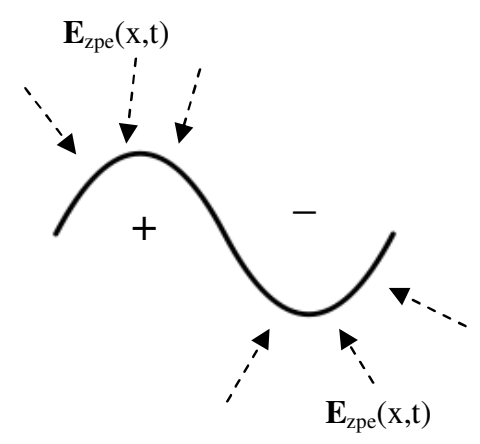

Figure 2

The electromagnetic field is modelled as a sum of Fourier modes[10] in three dimensions (eqn. 14). When quantised by the Uncertainty Principle it has a variance at zero photon count but no average. Each mode, flips randomly in time on the order of $\lambda / c$ and thus can be thought of as a dipole (figure 2), which in turn is acted upon by the random electric field from all the other zeropoint modes. 
$\mathbf{E}(\mathbf{r}, t)=i \sqrt{\frac{\hbar \omega}{2 V \varepsilon_{0}}} \sum_{\mathbf{k}}\left(a_{\mathbf{k}}(t) e^{i \mathbf{k} \cdot \mathbf{r}}-a_{\mathbf{k}}^{\dagger}(t) e^{-i \mathbf{k} \cdot \mathbf{r}}\right)$

$\mathbf{B}(\mathbf{r}, t)=i \sqrt{\frac{\hbar}{2 \omega V \varepsilon_{0}}} \sum_{\mathbf{k}} \mathbf{k} \times\left(a_{\mathbf{k}}(t) e^{i \mathbf{k} \cdot \mathbf{r}}-a_{\mathbf{k}}^{\dagger}(t) e^{-i \mathbf{k} \cdot \mathbf{r}}\right)$ eqn. 14

Polarisation is neglected for brevity.

The electromagnetic field is Lorentz invariant. This is begging the question as to whether the quantised modes can mechanically translate (the physics would be the same) and suffer a restoring force - a mechanical quantum harmonic oscillator built upon the zero-point mass-energy $m_{\omega \mathbf{1}}$ equivalent of the quantum harmonic oscillator of the electromagnetic field. One may worry that this leads to an infinityof-infinities of zeropoint terms as we sum each mode (or at least, that it is very large) but one may naively and roughly consider it thus: a mass-springdamper system being forced into motion by a random force of the zeropoint field and radiative damping[15] (proportional to $d^{3} x / d t^{3}$ ); we can take the complex Fourier (Laplace) transform:-

Where,

$$
X(s)=\frac{\underbrace{S_{\text {zpe }}(s)}_{\text {"Mass" }}}{\underbrace{}_{\text {"Damper" }} s^{2}-\zeta s^{3}}
$$

- The equivalent mass of the mode $m_{\omega \mathbf{1}}=\frac{1}{2} \frac{\hbar \omega_{1}}{c^{2}}$,

- The there isn't any restoring force as such but the stochastic ZPE perturbation $S_{z p e}(\omega)$.

- With damping (coupling to other modes),

$$
\xi=\frac{e^{2}}{6 \pi \varepsilon_{0} c^{3}}
$$

- The spectrum of the zeropoint electric field is the square root of the power density of the zeropoint field:-

$$
\begin{aligned}
S_{z p e}(\omega) & =\sqrt{\frac{\hbar}{8 \pi^{2} c^{3}} \int \omega^{3} d \omega} \\
& =\sqrt{\frac{\hbar}{8 \pi^{2} c^{3}}} \omega^{2}
\end{aligned}
$$

The average of the zeropoint electric field is zero (but it has a variance), the perturbation from it is a series of 1-D random walks (for each dimension). A moment's thought will have one realise that for equal probability of going "left" or "right" that this is just a binominal distribution and in the limit is Gaussian. The perturbation from the other modes just acts like a restoring force.
Differentiation of the position gives velocity and this can be performed in the s-domain by a multiplication by s. Moving from the complex Fourier domain to the just the Fourier domain, with the substitution $s_{\sigma \rightarrow 0}=\sigma+i \omega$, allows the plotting of "frequency response" of the system. The model for velocity in the frequency domain (via eqn. 15) becomes,

$$
V(\omega)=\frac{-i \sqrt{\frac{\hbar}{8 \pi^{2} c^{3}}} \omega^{3}}{-\frac{1}{2} \frac{\hbar \omega_{1}}{c^{2}} \omega^{2}-i \frac{e^{2}}{6 \pi \varepsilon_{0} c^{3}} \omega^{3}} \text { eqn. } 16
$$

This model is non-Relativistic but we shall "re-map" velocity with a reasonable procedure given in Appendix 2. However let us see where it gets us in our supposition that there are extra degrees of freedom to the zeropoint and get the form of $\hbar I\left(\omega_{1}, \omega\right)$ in eqn. 13 (hence the focus on $m_{\omega \mathbf{1}}$ "moving").

The Parseval- Plancherel[16] theorem in the frequency domain relates to the time domain thus,

$$
\int_{0}^{\infty}|v(t)|^{2} d t=\int_{0}^{\infty}|V(\omega)|^{2} d \omega \quad \text { eqn. } 17
$$

It is reasonable to compute the Root-Mean-Square value of the velocity over a time/frequency window as follows (where $\omega_{\mathrm{P}}$ is the Planck Frequency),

$$
\begin{aligned}
\left\langle v_{R M S}\right\rangle & \doteq \frac{1}{T} \sqrt{\int_{1 / \omega_{P}}^{T}|v(t)|^{2} d t} \\
& =\frac{1}{\omega_{P}-1 / T} \sqrt{\int_{1 / T}^{\omega_{P}}|V(\omega)|^{2} d \omega}
\end{aligned}
$$

Appendix 2 performs the integral in the frequency domain of eqn. 16 by a Bodé plot (magnitude vs. frequency) for low, intermediate and high $m_{\omega \mathbf{1}}$ to match our assertion that the zeropoint has higher degrees of freedom and eqn. 13 is of the form:

$$
H_{z p e}=\frac{1}{2}\left(\hbar \omega_{1}+\gamma \hbar \omega_{1}\right) \quad \text { eqn. } 19
$$

And with $\gamma$ in the ballpark of $10^{9}$, per each spatial dimension, this supplies the necessary $10^{9}$ or so for the factor to have,

$$
U^{-3} \kappa^{3} \rho_{Q F T}=\rho_{c c}
$$

This completes the proof that there is a direct relation between the zeropoint of quantum field theories and the observed vacuum constant/Dark Energy of Astronomy/Cosmology, whereas before there was a ghastly chasm of at least $10^{120}$ orders of magnitude. A more accurate and refined model for 
these extra degrees of motion of the zeropoint may make that relation precise.

\section{$\underline{\text { Conclusion }}$}

In looking at the theoretical underpinnings of a novel propellant-less electromagnetic propulsion engine, the author has been forced to look at the reality of the zeropoint. What emerges is that the zeropoint field appears to be a superfluid or supersolid, with the interaction between the modes forming this solid giving the system more degrees of freedom, such that the zeropoint energy is some $10^{9}$ higher than currently calculated.

Fortuitously a direct relation between the zeropoint of quantum field theories and the observed vacuum constant (or Dark Energy) of Astronomy and Cosmology is obtained by a polynomial expansion of the stress energy tensor in the Einstein constant to $3^{\text {rd }}$ order and a Taylor expansion of the said tensor too to $2^{\text {nd }}$ order. The relationship is almost exact pending a revision of our rough model of the extra degrees of freedom previously mentioned. The $10^{120}$ orders of magnitude problem has been solved.

The well-known Pauli theory on zeropoint says that the mass-less electromagnetic photon must have the dominant zeropoint over massive fermion fields and so we concentrated on the former to establish a relation between it and Dark Energy by a model and modification to the stress-energy tensor: it simply isn't correct to attempt to put the zero photon count of the electromagnetic zeropoint into the tensor at zeroth order.

The numerology of the model seems arbitrary but in its favour it is physical and not merely mathematical trickery: it reasonably asserts that there is interaction between the modes of the zeropoint and the model drops zeropoint energy from the stress-energy tensor in the zeroth order (and concerns over why it doesn't severely gravitate or expand the universe) and introduces it as a fluctuation in the second $\operatorname{order}(\Delta \omega)^{2}$ which is correct and looks promising, as the zeropoint has a variance but no average.

Appendix 1 - Dimensional Analysis of " $U$ " and its interpretation

Dimensional analysis of $\mathrm{U}$, the factor introduced into eqn. 12 means it has units of Newtons ${ }^{-1}$ or meters/Joules. This is very similar to the spring constant (an inverse spring constant in case of $U$ ),

$$
d F_{x}=K_{x} d x
$$

If we extend this to two dimensions:

$$
d F_{x} \times d F_{y}=\left(K_{x} \otimes K_{y}\right) d x \times d y
$$

The tensor $\left(K_{x} \otimes K_{y}\right)$ formed provides notions of a flexural rigidity tensor that describes the deforming of area $d A=d x \times d y$. Thus if $\mathrm{U}^{-2}$ had units $[\mathrm{N}]^{2} /[\mathrm{M}]^{2}$ in the $3^{\text {rd }}$ term in eqn. 12 , it would mean it is some kind of flexural rigidity but taken together with the $3^{\text {rd }}$ order in $\kappa$ (the Einstein-Newton constant) it becomes (maybe) just a modified Einstein-Newton constant,

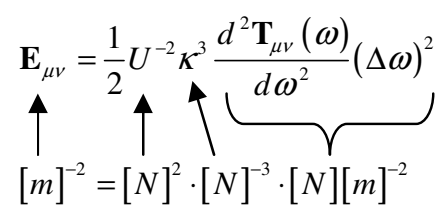

(eqn. 11)

compare with

$$
\begin{aligned}
& \frac{1}{d A}=K^{2} \frac{1}{d F_{x} \times d F_{y}} \\
& {[m]^{-2}=[N / m]^{2} \cdot[N]^{-2}}
\end{aligned}
$$

The first option with $U$ as a flexural rigidity constant suggests that eqn. 12 has something to do with deforming an infinitesimal area element of spacetime against an intrinsic pressure and that this is somehow related to zeropoint fluctuations. This may have something to do with the "elasticity of spacetime" too, as Sakharov put it[17]; both effects seem related to the same source - $\rho_{Q F T}$.

Yet, mundanely, we intended to relate $\rho_{Q F T}$ to $\rho_{c c}$ thus, $U^{-3} \kappa^{3} \rho_{Q F T}=\rho_{c c}$. Also $\rho_{Q F T}$ was increased (non-arbitrarily or trivially but given extra degrees of freedom) to make this true. U, can pick up this increase or just maintain the units and do nothing. So,

$$
G=\frac{c^{4}}{8 \pi} U \cdot \sqrt[3]{\frac{\rho_{c c, \text { measured }}}{\rho_{Q F T}\left(\hbar, \pi, \omega_{\text {Planck }}\right)}}
$$

The hypothesis in this paper was built to relate $\rho_{c c, \text { measured }}$ directly to $\rho_{Q F T}\left(\hbar, \pi, \omega_{\text {Planck }}\right)$. One wonders if $\mathrm{G}$ is a combined fundamental constant of nature and mathematics.

Appendix 2 - Estimation of integral eqn. 17 over three different effective mass ranges

Model eqn. 16 is non-Relativistic. The Laplace transform doesn't work well with non-linear expressions, so it is not easy to introduce gamma into the mass term of eqn. 15(eqn. 16); however the frequency domain and the Parseval-Plancherel[16] 
theorem offers an enticing method to solve our problem. Setting up a differential equation solver, as per the Matlab ${ }^{\circledR}$ system is prohibitive too, for the very small step size needed to compute effects on the order of the Planck Time, when dealing with the gamut of electromagnetic frequencies almost down to $\mathrm{OHz}$. Thus we shall persist with the frequency domain approach and remap impermissible Newtonian speeds of the model to Relativistic speeds by the observation that the total energy of the system to $1^{\text {st }}$ order in $\mathrm{v}^{2} / \mathrm{c}^{2}$ gives the Newtonian kinetic energy (and the rest mass),

$$
\begin{gathered}
E_{0}+E_{0}\left(\frac{v_{1}^{2}}{2 c^{2}}\right) \underset{v_{1} \text { to } v}{\longrightarrow} \frac{E_{0}}{\sqrt{1-\frac{v^{2}}{c^{2}}}} \\
\Rightarrow\left(1+\frac{v_{1}^{2}}{2 c^{2}}\right)=\frac{1}{\sqrt{1-\frac{v^{2}}{c^{2}}}} \\
\Rightarrow v=c \sqrt{1-\frac{4 c^{4}}{\left(2 c^{2}+v_{1}^{2}\right)^{2}}}
\end{gathered}
$$

The model is implemented with Matlab and is in the next appendix. A typical output of the logarithm of the speed of the oscillator vs. logarithm of frequency up to the Planck Frequency looks like this:-

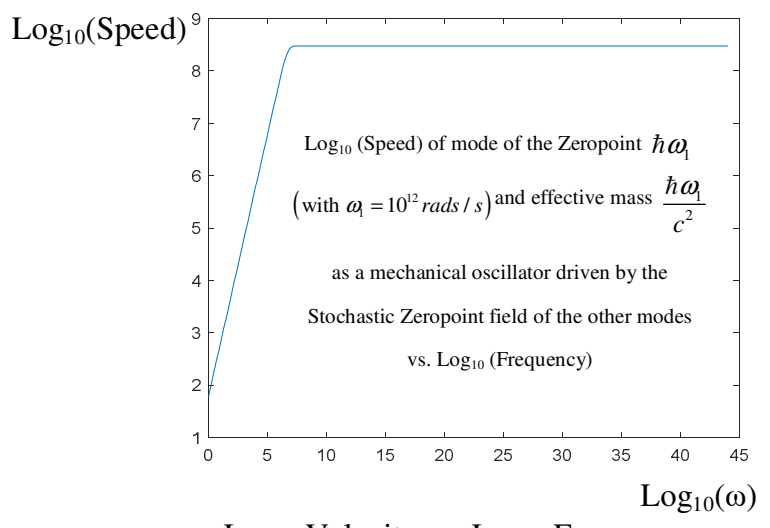

$\underline{\log _{10}} \underline{\text { Velocity vs. } \log _{\underline{10}} \text { Frequency }}$

This is displaying a reluctance to "move" at lower frequencies, from the stochastic excitation from the electric field of the other modes, due to the effective mass of the mode. However, once this is overcome, the speed tends to the speed of light.

Application of the Parseval-Plancherel theorem and eqn. 18 to calculate the RMS of the speed is just the area under the graph (absolute value not the logarithm) and by inspection, one can see that this value is less than light-speed. This limit is part increase in inertia and "drag" by the LorentzAbrahams-Dirac force coupling to other modes and partitioning the kinetic energy. The form of eqn. 19 and hence eqn. 13 is correct:

$$
\begin{aligned}
H_{z p e} & =\frac{1}{2}\left(\hbar \omega_{1}+\gamma \hbar \omega_{1}\right) \\
\text { Or, } \quad & =\frac{1}{2}\left(\hbar \omega_{1}+\frac{\hbar \omega_{1}}{\sqrt{1-\frac{v^{2}}{c^{2}}}}\right)
\end{aligned}
$$

And it is suggested that $\gamma \simeq 10^{9}$ to match the value of the energy of the vacuum of QFTs to the Cosmological Constant. If the speed of light ${ }^{* *}$ is defined to be $299,792,458 \mathrm{~m} / \mathrm{s}$, it would require our velocity (for that gamma) to be defined to precision better than 1 part in $10^{-18}$, hence the velocity would be,

$$
299,792,457.999,999,999,8(5) \mathrm{m} / \mathrm{s}
$$

The model for verification requires more precision than Metrology can supply at the moment ${ }^{\dagger \dagger}$, however that doesn't rule it out as potentially untestable but plausible. The notion that the zeropoint has extra degrees of freedom still stands.

It is worth taking a look at the oscillation of other modes, that is with $\hbar \omega_{1}$ and hence $\omega_{1}$ with other values. Below shows $\omega_{1}=10^{6} \cdots 10^{6+3 x} \cdots 10^{36} \mathrm{rad} / \mathrm{s}$ and then $\omega_{1}=10^{40}, 10^{41}, 10^{42} \mathrm{rad} / \mathrm{s}$,

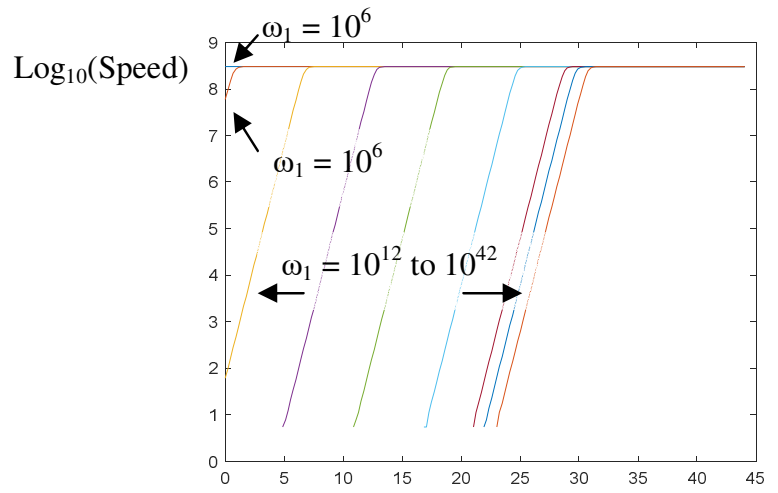

$\log _{10}(\omega)$

In this case the gammas $\left(\omega_{1}\right)$ would not be constant at $10^{9}$ but the weighted sum provided by eqn. 13 would affect $\rho_{Q F T}$ to give $U^{-3} \kappa^{3} \rho_{Q F T}=\rho_{c c}$.

\footnotetext{
** The General Conference on Weights and Measures/International System of Units

${ }^{\dagger \dagger}$ https://physicsworld.com/a/fundamentalconstant-measured-at-highest-precision-yet/
} 
$-7-$

Appendix 3 - Matlab model to generate Bodé plots

One simply runs the programme as $\operatorname{Bode}(\omega)$ and the numerical value of the mode in question in $\mathrm{rad} / \mathrm{s}$.

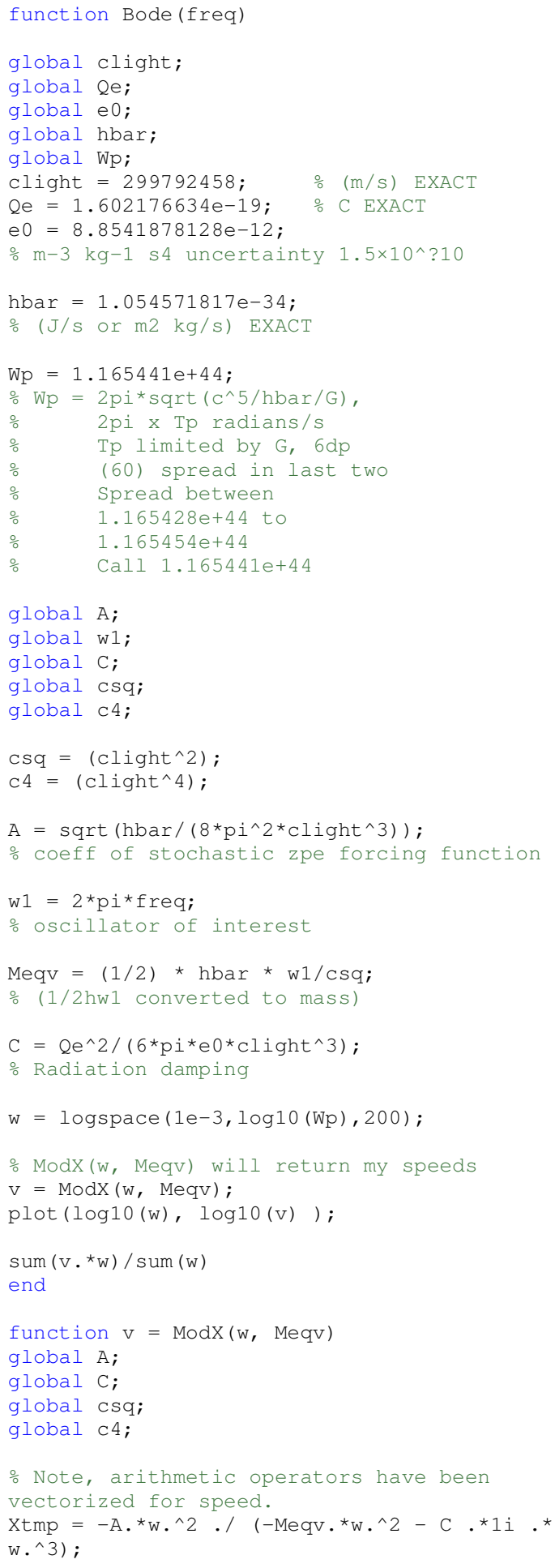

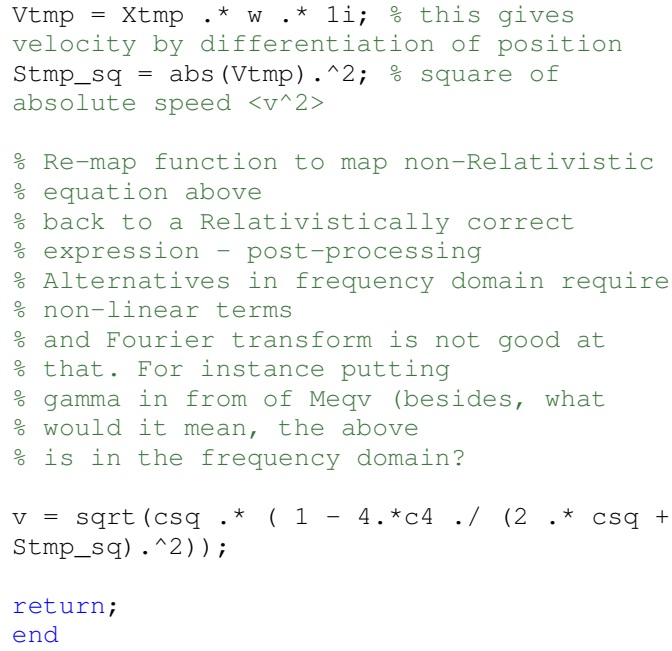

To obtain the plot with multiple Bodé plots, type "hold on" before running the program again.

\section{$\underline{\text { References }}$}

1. Cornwall, R.O., A Mechanism for Propulsion without the Reactive Ejection of Matter or Energy. Preprints, 2019 https://www.academia.edu/38062548/A_ Mechanism_for_Propulsion_without_The _Reactive_Ejection_of_Matter_or_Energy

2. Feynman Leighton Sands, The Feynman Lectures on Physics. Addison-Wesley, Reading, Massachusetts. Vol. 21989.

3. Comay, Exposing "hidden momentum". Am. J. Phys., 1996. 64(8).

4. Graham Lahoz, Observation of static electromagnetic angular momentum in vacuo. Nature, 1980. 285(154).

5. Hernandez, A., The Feynman paradox revisited. Eur. J. Phys., 1981. 2: p. 168170.

6. Kirk, T. On the Definition of "Hidden" Momentum. 2017; Available from: http://physics.princeton.edu/ mcdonald/ex amples/hiddendef.pdf

7. Cornwall, R.O., The Energetics of the an ExB propulsor that avoids the Hidden Momentum pitfall. Preprints, 2019 https://www.academia.edu/41062130/The Energetics_of_the_an_ExB_propulsor_th at_avoids_the_Hidden_Momentum_pitfall 
8. Cornwall, R.O., Dissipation of Momenergy to a Bose Gas by An Electromagnetic Propulsion Device. Preprints, 2020 https://www.academia.edu/41900817/Diss ipation_of_Momenergy_to_a_Bose_Gas by_An_Electromagnetic_Propulsion_Devi ce

9. Cornwall, R.O., Candidates for EM propulsion - slide presentation. Preprints, 2021 https://www.academia.edu/49712716/Micr osoft_PowerPoint_APEC

10. Landau Lifshitz, A Course in Theoretical Physics: Quantum Electrodynamics. Butterworth-Heinemann. Vol. 4. 1984.

11. Baez, J. What's the Energy Density of the Vacuum? 2011; Available from: http://math.ucr.edu/home/baez/vacuum.ht $\underline{\mathrm{ml}}$

12. Zee, A., Quantum Field Theory in a Nutshell. 2003: Princeton U. Press.

13. Pauli, W., Pauli Lectures on Physics: Vol 6, Selected Topics in Field Quantization. 1971(1951): MIT Press: Cambridge, MA, USA.

14. Visser, M., Lorentz invariance and the zero-point stress-energy tensor. Arxiv.org preprint, 2018(arXiv:1610.07264).

15. Jackson, J.D., Classical Electrodynamics. 2nd ed. 1975: Wiley.

16. Kreyzig, E., Advanced Engineering Mathematics. Wiley Interscience. 2000.

17. Misner, C.T., K.; Wheeler, J., Gravitation. 21st ed. 1998: W. H. Freeman and Co. 